\title{
Gribov gap equation at finite temperature
}

\author{
Fabrizio Canfora ${ }^{1,2, a}$, Pablo Pais ${ }^{1,2, b}$, Patricio Salgado-Rebolledo ${ }^{1,3,4, \mathrm{c}}$ \\ ${ }^{1}$ Centro de Estudios Científicos (CECS), Casilla, 1469 Valdivia, Chile \\ 2 Universidad Andrés Bello, Av. República 440, Santiago, Chile \\ ${ }^{3}$ Departamento de Física, Universidad de Concepción, Casilla 160-C, Concepción, Chile \\ ${ }^{4}$ Physique Théorique et Mathématique, Université Libre de Bruxelles and International Solvay Insitutes, Campus Plaine C.P. 231, \\ 1050 Bruxelles, Belgium
}

Received: 5 December 2013 / Accepted: 2 April 2014 / Published online: 20 May 2014

(C) The Author(s) 2014. This article is published with open access at Springerlink.com

\begin{abstract}
In this paper the Gribov gap equation at finite temperature is analyzed. The solutions of the gap equation (which depend explicitly on the temperature) determine the structure of the gluon propagator within the semi-classical Gribov approach. The present analysis is consistent with the standard confinement scenario for low temperatures, while for high enough temperatures, deconfinement takes place and a free gluon propagator is obtained. An intermediate regime in between the confined and free phases can be read off from the resulting gluon propagator, which appears to be closely related to partial deconfinement.
\end{abstract}

\section{Introduction}

One of the most characteristic features of QCD is asymptotic freedom $[1,2]$, which allows one to perform the standard perturbative analysis in the ultraviolet regime. On the other hand, the infrared regime of the theory is not well understood yet from the analytical point of view, as the running coupling is large for low energies. Indeed, color confinement is one of the main open problems in theoretical physics.

The standard perturbative approach to avoid overcounting of gauge equivalent configurations in Yang-Mills (YM) theory is to introduce a gauge fixing condition in the functional integral (the Landau gauge will be considered in the following). However, as Gribov pointed out [3], the Landau gauge condition does not fix the gauge completely. Shortly after, Singer showed that, due to the non-trivial nature of the fiber bundle structure of YM-theory, any true gauge condition presents this obstruction [4] (see also [5]). The presence of Gribov copies close to the identity induces the existence

\footnotetext{
a e-mail: canfora@cecs.cl

be-mail: pais@cecs.cl

ce-mail: pasalgado@udec.cl
}

of non-trivial zero modes of the Faddeev-Popov operator, which make the path integral ill defined. Even when perturbation theory around vacuum is not affected by Gribov ambiguity when YM-theory is defined over a flat space-time ${ }^{1}$ with trivial topology [10], Gribov copies have to be taken into account when considering more general cases [11] or when non-perturbative phenomena are studied.

The most effective method to eliminate Gribov copies (proposed by Gribov himself in [3] and refined in [12-14]) corresponds to restricting the path integral to the so-called Gribov region, which is the region in the functional space of gauge potentials over which the Faddeev-Popov operator is positive definite. In [12] Dell' Antonio and Zwanziger showed that all the orbits of the theory intersect the Gribov region, indicating that no physical information is lost when implementing this restriction. Even though this region still contains copies with non-trivial winding number [15], this restriction has remarkable effects. In fact, the gluon propagator is suppressed in the infrared and the ghost propagator is enhanced, which has opened a way to understand color confinement $[10,16]$. A local and renormalizable effective action for YM-theory whose dynamics is restricted to the Gribov horizon and that yields the same results for the field propagators was constructed in [13,14,17-20] by adding extra fields to the action. Later, an improved action was proposed by considering suitable condensates, which leads to propagators and glueball masses in agreement with the lattice data [21-23]. With the same action, one can also solve the old problem of the Casimir energy in the MIT-bag model [24].

Even though it is an experimental fact that quarks and gluons are confined and color charged states are unobservable as asymptotic states at low temperatures, it is expected that

\footnotetext{
${ }^{1}$ In the curved case, the pattern of appearance of Gribov copies can be considerably more complicated: see in particular [6-9]. Therefore, only the flat case will be considered.
} 
at high temperatures $\left(T_{c} \sim 150-200 \mathrm{MeV}\right)$ they become free $[25,26]$. Such a phase transition from confinement to quark-gluon plasma (QGP) should be described within the framework of finite-temperature field theory allowing a better understanding of natural scenarios as the early universe or compact star physics [26-28]. The high-temperature sector for the theory corresponds to the perturbative region, in which gluons are physical states. In this regime it is necessary to include thermal loop corrections, which may yield a dynamical thermal mass generation for the gauge fields [26]. In particular, the hard thermal loop approximation cancels infrared divergences coming from the Matsubara frequencies, allowing one to study plasma oscillations. On the other hand, lattice QCD allows one to handle non-perturbative phenomena at finite temperature, such as phase transitions. The critical temperature for QGP has been subject of several studies as well as its relation with the energy scale in the context of quenched QCD [29,30]. Moreover, some recent analyses (see, in particular [31-34], and references therein) strongly support the existence of an intermediate regime which lies in between the confined phase and the free phase. Within this regime, some features of the confined phase coexist with the high-temperature plasma phase. Although it is not clear yet whether this intermediate phase corresponds to a phase transition or to a cross-over, it can be safely assumed that such an intermediate regime does appear.

In this paper we will analyze the semi-classical Gribov approach to QCD at finite temperature ${ }^{2}$ extending the pioneering works $[35,36]$. To this aim, the finite-temperature theory at one loop will be restricted to the Gribov region and the existence of phase transitions from confinement to gluon plasma will be analyzed. Since gluon deconfinement is associated to the presence of dynamical thermal mass [37], the contribution of thermal loops to the finite-temperature analysis cannot be ignored when implementing this restriction. The semi-classical Gribov analysis shed considerable light on the non-perturbative behavior of the gluon propagator. Indeed, at zero temperature, the existence of a non-trivial solution to the Gribov gap equation implies that the gluon propagator has imaginary poles and consequently gluons do not belong to the physical spectrum. Hence, there are two important requirements that the finite-temperature gap equation must satisfy. Firstly, the finite-temperature gap equation should have, when the temperature is low enough, solutions close to the zero-temperature one, describing confined glu-

\footnotetext{
${ }^{2}$ A remark on the terminology: in the following we will denote by "critical temperatures" the temperatures which correspond to changes of the qualitative behavior of the solutions of the finite-temperature Gribov gap equation. Although the present analysis by itself is not enough to prove rigorously the appearance of a phase transition (since suitable order parameters should be identified and analyzed), we think that this terminology is useful to emphasize the sharp differences in the behavior of the Gribov gluon propagator as the temperature changes.
}

ons. Secondly, when the temperature is high enough, the finite-temperature gap equation should describe propagating gluons. Although it is not easy to satisfy both conditions $[35,36]$, here we will show that not only they can be satisfied, but also that the finite-temperature gap equation discloses the presence of a new regime in between the confined and free regimes, which appears to be closely related to the intermediate regime mentioned before.

The paper is organized as follows: In Sect. 2 the quantization of YM-theory and the semi-classical Gribov approach at zero temperature are briefly reviewed following the lines of [10]. In Sect. 3 the main considerations for the one-loop finite-temperature analysis are exposed, and a thermal gap equation is derived. Section 4 is devoted to the numerical study of a phase transition taking into account a different possible temperature dependence for the QCD running coupling. Finally, in Sect. 5, we discuss the results and compare them with the known literature.

Note added: recently, Ref. [38] was posted on arXiv, where this problem is also studied by a different approach.

\section{Semi-classical Gribov approach to QCD}

In this section we will briefly review the semi-classical procedure to restrict the path integral formulation of YM-theory to the Gribov region following the lines of $[3,10]$.

The action functional for $S U(N)$ Euclidean YM-theory is given by

$S_{\mathrm{EYM}}[A]=\frac{1}{4 g_{0}^{2}} \int \mathrm{d}^{4} x F_{\mu \nu}^{a} F_{a}^{\mu \nu}$,

where $g_{0}$ is the coupling constant, $F_{\mu \nu}^{a}=\partial_{\mu} A_{\nu}^{a}-\partial_{\nu} A_{\mu}^{a}+$ $f^{a}{ }_{b c} A_{\mu}^{b} A_{v}^{c}$ is the field strength tensor associated to the fourpotential $A_{\mu}=A_{\mu}^{a} T_{a}$, and the $T_{a}$ are the anti-hermitian generators of the $\operatorname{su}(N)$ algebra

$\left[T_{a}, T_{b}\right]=f_{a b}^{c} T_{c}$,

with $f_{b c}^{a}$ the $s u(N)$ structure constants. The action (1) is invariant under $S U(N)$ gauge transformations

$A_{\mu} \rightarrow A_{\mu}^{\prime}=h^{\dagger}\left(A_{\mu}+\partial_{\mu}\right) h, h \in S U(N)$.

The quantum theory can be constructed by defining the Feynman path integral. In order to sum only over inequivalent configurations, a gauge fixing condition must be implemented via the Faddeev-Popov trick. In the Landau gauge $\partial^{\mu} A_{\mu}=0$, the gauge fixed path integral has the standard form [40]

$Z=\mathcal{N} \int D A D c D \bar{c} \delta\left(\partial^{\mu} A_{\mu}\right) \operatorname{det}(\mathcal{M}) \exp \left(-S_{\mathrm{EYM}}\right)$, 
where $\mathcal{N}$ is a normalization, and $\mathcal{M}$ is the Faddeev-Popov operator for the Landau gauge condition:

$\mathcal{M}_{b}^{a}=-\partial^{\mu}\left(D_{\mu}\right)_{b}^{a}$,

with $\left(D_{\mu}\right)_{b}^{a}=\delta_{b}^{a} \partial_{\mu}-f_{b c}^{a} A_{\mu}^{c}$ the covariant derivative in the adjoint representation.

Due to the presence of Gribov copies [3], however, the expression (2) is ill defined. To avoid zero modes of the Faddeev-Popov operator and eliminate copies, Gribov proposed to restrict the path integral to the so-called Gribov region $C_{0}$, which corresponds to the region in the functional space of gauge potentials over which the Faddeev-Popov operator is positive definite,

$C_{0} \equiv\left\{A_{\mu}, \partial^{\mu} A_{\mu}=0 \mid \operatorname{det} \mathcal{M}>0\right\}$.

The restriction of (2) to the Gribov region can be implemented by redefining the generating functional as

$$
\begin{aligned}
Z_{G}= & \mathcal{N} \int D A D \bar{c} D c \delta\left(\partial^{\mu} A_{\mu}\right) \\
& \times \operatorname{det}(\mathcal{M}) \exp \left(-S_{\mathrm{YM}}\right) \mathcal{V}\left(C_{0}\right),
\end{aligned}
$$

where the factor $\mathcal{V}\left(C_{0}\right)$ ensures that the integration is performed only over $C_{0}$. In order to characterize $\mathcal{V}\left(C_{0}\right)$, we look at the connected two-point ghost function generated by (2):

$$
\begin{aligned}
\left\langle\bar{c}^{a}(x) c^{b}(y)\right\rangle= & \mathcal{N} \int D A \delta\left(\partial^{\mu} A_{\mu}\right) \exp \left(-S_{\mathrm{YM}}\right) \\
& \times \operatorname{det}(\mathcal{M})\left(\mathcal{M}^{-1}(x, y)\right)^{a b} .
\end{aligned}
$$

Singularities in (6) correspond to zero modes of the FaddeevPopov operator, i.e. infinitesimal Gribov copies. In the momentum representation, singularities different from $k^{2}=$ 0 imply that $\mathcal{M}(x, y)$ can become negative definite, and therefore it is evaluated outside the Gribov horizon. The factor $\mathcal{V}\left(C_{0}\right)$ must be such that this kind of singularities is not present. This is known as the "no-pole condition".

The standard connected ghost two-point function (6) can be put in the form

$$
\begin{aligned}
\left\langle\bar{c}^{a}(x) c^{b}(y)\right\rangle= & \mathcal{N} \int D A D c D \bar{c} \delta\left(\partial^{\mu} A_{\mu}\right) \\
& \times \exp \left(-S_{\mathrm{YM}}\right)\left\langle\bar{c}^{a}(x) c^{b}(y)\right\rangle_{A},
\end{aligned}
$$

with $\left\langle\bar{c}^{a}(x) c^{b}(y)\right\rangle_{A}$ the connected ghost two-point function with $A_{\mu}^{a}$ playing the role of an external field. To second order in perturbation theory this can be written in momentum space as

$$
\left\langle\bar{c}^{a} c_{a}\right\rangle_{k ; A}=\frac{1}{k^{2}}(1+\sigma(k, A)) \approx \frac{1}{k^{2}} \frac{1}{(1-\sigma(k, A))},
$$

where

$$
\begin{aligned}
\sigma(k, A)= & \frac{N k^{\mu} k^{\nu}}{3\left(N^{2}-1\right) k^{2}} \frac{1}{V} \sum_{q} \frac{A^{a \lambda}(-q) A_{a \lambda}(q)}{(k-q)^{2}} \\
& \times\left(\delta_{\mu \nu}-\frac{q_{\mu} q_{\nu}}{q^{2}}\right),
\end{aligned}
$$

and $V$ stands for the four-dimensional volume of the Euclidean space-time. Since $A_{\mu}^{a}(-q) A_{a v}(q)$ is a decreasing function of $q^{2}, \sigma(k, A)$ decreases as $k^{2}$ increases and the no-pole condition can be stated as

$\sigma(0, A)=\frac{1}{4} \frac{N}{N^{2}-1} \frac{1}{V} \sum_{q} \frac{1}{q^{2}} A_{\mu}^{a}(-q) A_{a}^{\mu}(q)<1$.

Hence, the factor $\mathcal{V}\left(C_{0}\right)$ needed in (5) to restrict path integrals to the Gribov horizon is given by $\mathcal{V}\left(C_{0}\right)=\Theta(1-$ $\sigma(0, A))$, where $\Theta(x)=\frac{1}{2 \pi i} \int_{-i \infty+\varepsilon}^{i \infty+\varepsilon} \mathrm{d} \eta \frac{\mathrm{e}^{\eta x}}{\eta}$ is the Heaviside step function. Implementing this factor in $Z_{G}$, the quadratic part of the path integral in the field $A_{\mu}$ can be put in the form

$$
\begin{aligned}
Z_{G}^{\text {quad }}= & \mathcal{N} \int \frac{\mathrm{d} \eta}{2 \pi i} \mathrm{e}^{f(\eta)}, \quad f(\eta)=\eta-\ln \eta \\
& -\frac{3}{2}\left(N^{2}-1\right) \sum_{q} \ln \left(q^{2}+\frac{\eta N g_{0}^{2}}{N^{2}-1} \frac{1}{2 V} \frac{1}{q^{2}}\right)
\end{aligned}
$$

Using the steepest descent (saddle point) method, (11) can be approximated by $Z_{G}^{\text {quad }} \approx \mathrm{e}^{f\left(\eta_{0}\right)}$, where $\eta_{0}$ satisfies the minimum condition $f^{\prime}\left(\eta_{0}\right)=0$. Defining the Gribov parameter $\gamma^{4}=\frac{\eta_{0} N g_{0}^{2}}{N^{2}-1} \frac{1}{2 V}$, the minimum condition leads to the gap equation

$1-\frac{N g_{0}^{2}}{\gamma^{4}\left(N^{2}-1\right) 2 V}-\frac{3 N g_{0}^{2}}{4 V} \sum_{q} \frac{1}{q^{4}+\gamma^{4}}=0$.

The solution of this equation in the infinite volume limit $V \rightarrow$ $\infty$ is given by $\gamma^{2}=\Lambda^{2} \mathrm{e}^{-\frac{64 \pi^{2}}{3 N g_{0}^{2}}}$, where $\Lambda$ is the ultraviolet cutoff, and it leads to a confining gauge propagator [10]

$D_{\mu \nu}^{a b}(q)=\delta^{a b} g_{0}^{2} \frac{q^{2}}{q^{4}+\gamma^{4}}\left(\delta_{\mu \nu}-\frac{q_{\mu} q_{\nu}}{q^{2}}\right)$.

For large $q$, (13) reduces to the standard perturbative result [40]. In the infrared, however, the gluon propagator is suppressed, as it displays imaginary poles. In other words, since $D_{\mu \nu}^{a b}(q)$ has a positivity violating Källén-Lehmann representation $[39,40]$, gluons cannot be considered as part of the physical spectrum and the propagator (13) is interpreted as confining.

Replacing (13) in (9) leads to the following behavior for the ghost propagators (8), in the infrared limit:

$\left\langle\bar{c}^{a} c_{a}\right\rangle_{q ; A} \underset{q \rightarrow 0}{\longrightarrow} \frac{128 \pi \gamma^{2}}{3 N g_{0}^{2}} \frac{1}{q^{4}}$, 
which means that the ghost propagator is not free-like, but enhanced for $q \rightarrow 0$.

\section{Finite-temperature analysis}

Finite-temperature YM-theory can be studied using the imaginary time formalism $[25,41]$, which relates the corresponding quantum field theory generating functional with a quantum statistical partition function through a compactification of the temporal coordinate. In this formalism, the period of the compactified time is associated with the inverse of the temperature of a thermal bath, and the partition function can be written as

$$
Z=\int D A \exp \left(\frac{1}{4 g_{0}^{2}} \int_{0}^{\frac{1}{T}} d \tau \int d^{3} x F_{\mu \nu}^{a} F_{a}^{\mu \nu}\right) .
$$

Since the temporal integration limits 0 and $T^{-1}$ are identified, when passing to momentum space, temperature dependent fields are expanded in a Fourier series over discrete Matsubara frequencies $\omega_{n}$.

$$
\begin{aligned}
& \varphi(\tau, \mathbf{x})=T \sum_{\substack{n=-\infty \\
\omega_{n}}}^{\infty} \int \frac{\mathrm{d}^{3} q}{(2 \pi)^{3}} \mathrm{e}^{-i\left(\omega_{n} \tau+\mathbf{q} \cdot \mathbf{x}\right)} \varphi\left(\omega_{n}, \mathbf{q}\right), \\
&
\end{aligned}
$$

\subsection{Dynamical thermal mass}

When implementing the gauge fixing, the finite-temperature formalism must be applied to the generating functional (2), where the Euclidean action has to be written as a local functional for ghost and gauge fields and perturbation theory can be applied. For gluons, when considering one-loop corrections, the resumed gauge propagator in the Landau gauge takes the form [26]

$D_{\mu \nu}^{a b}(q)=g^{2} \delta^{a b}\left(\frac{P_{\mu \nu}^{T}(q)}{q^{2}+\Pi_{T}(q)}+\frac{P_{\mu \nu}^{L}(q)}{q^{2}+\Pi_{L}(q)}\right)$,

where $g$ is the running coupling and

$P_{\mu \nu}^{T}(q)=\delta_{\mu}^{i} \delta_{\nu}^{j}\left(\delta_{i j}-\frac{q_{i} q_{j}}{\mathbf{q}^{2}}\right)$,

$P_{\mu \nu}^{L}(q)=\delta_{\mu \nu}-\frac{q_{\mu} q_{\nu}}{q^{2}}-P_{\mu \nu}^{T}(q)$

are transverse projectors orthogonal to each other, $\left(P_{\mu \nu}^{T} q^{\nu}=\right.$ $\left.P_{\mu \nu}^{L} q^{\nu}=0, \delta^{\rho \sigma} P_{\mu \rho}^{T} P_{\sigma \nu}^{L}=0\right)$, and $\Pi_{T}(q), \Pi_{L}(q)$ are the components of the self-energy $\Pi_{\mu \nu}$ along the projectors (18)

$\Pi_{\mu \nu}(q)=P_{\mu \nu}^{T}(q) \Pi_{T}(q)+P_{\mu \nu}^{L}(q) \Pi_{L}(q)$.

In the plasma region, where $\omega_{n}>>|\mathbf{q}|$, the self-energy components $\Pi_{T}(q), \Pi_{L}(q)$ are given, in the hard thermal loop approximation, by
$\Pi_{T}(q)=\Pi_{L}(q) \approx \frac{N g^{2} T^{2}}{9}$,

which means that, in a hot plasma, gauge fields acquire an effective thermal mass [26]

$m_{p l}^{2}=\frac{N g^{2} T^{2}}{9}$.

In this case the gauge propagator (17) takes the form

$D_{\mu \nu}^{a b}(q)=\frac{g^{2} \delta^{a b}}{q^{2}+m_{p l}^{2}}\left(\delta_{\mu \nu}-\frac{q_{\mu} q_{\nu}}{q^{2}}\right)$.

It is worth noting that ghost fields do not acquire a thermal mass [25], which implies that the no-pole condition (10) has no extra terms when one-loop corrections are considered. However, the expression for the gap equation will be modified by the presence of the effective thermal mass (21), as we will see below.

\subsection{Gluon propagator in the presence of dynamical mass}

The effect of a dynamical mass $m$ in the semi-classical Gribov approach discussed in Sect. 2 can be obtained by adding a term of the form $m^{2} A_{\mu} A^{\mu}$ to the quadratic action in (11). This approach was studied in [42] and modifies the gap equation (12) as

$1-\frac{3 g^{2}}{\gamma^{4}\left(N^{2}-1\right) 2 V}-\frac{3 N g^{2}}{4 V} \sum_{q} \frac{1}{q^{4}+m^{2} q^{2}+\gamma^{4}}=0$.

The solution of this equation, if it exists, defines a massive (partially) confining gauge propagator

$\bar{D}_{\mu \nu}^{a b}(q)=\delta^{a b} g^{2} \frac{q^{2}}{q^{4}+m^{2} q^{2}+\gamma^{4}}\left(\delta_{\mu \nu}-\frac{q_{\mu} q_{\nu}}{q^{2}}\right)$.

The confining character of this propagator relies on the presence of imaginary poles, which violates positivity of the spectral density function of the Källén-Lehmann representation $[39,40]$, indicating that it describes non-physical excitations. However, the presence of a dynamical mass $m$ allows the possibility for the propagator (24) to acquire a physical degree of freedom. In fact, the poles of (24) are given by

$z_{ \pm}=\frac{1}{2}\left(-m^{2} \pm \sqrt{m^{4}-4 \gamma^{4}}\right)$.

Hence, for $m^{2} \geq 2 \gamma^{2}$ the propagator $\bar{D}_{\mu \nu}^{a b}(q)$ can describe physical particles. Writing (24) in the form

$$
\begin{aligned}
\bar{D}_{\mu \nu}^{a b}(q)= & \delta^{a b} \frac{g^{2}}{\sqrt{m^{4}-4 \gamma^{4}}}\left[\frac{z_{+}}{\left(q^{2}-z_{+}\right)}-\frac{z_{-}}{\left(q^{2}-z_{-}\right)}\right] \\
& \times\left(\delta_{\mu \nu}-\frac{q_{\mu} q_{\nu}}{q^{2}}\right),
\end{aligned}
$$


we can see that the propagator splits into two terms with opposite residue sign, indicating that the gluon field $A_{\mu}$ has only one physical degree of freedom.

In general, if $m$ is a function of some physical parameter, we can distinguish three scenarios for the behavior of the propagator.

- For $m^{2}<2 \gamma^{2}$ both poles of (24) are complex, indicating that there are no propagating gluonic degrees of freedom (confined phase).

- For $m^{2} \geq 2 \gamma^{2}$ only one of the two gluonic degrees of freedom is physical (partially deconfined phase). Hence, if this regime appears (as will be shown in the following, it does) it shows qualitative characteristics both of the confined phase and of the deconfined phase.

- If there is no solution for the gap equation, the only consistent choice for the Gribov mass parameter is $\gamma=0$, leading to a free gluon propagator (deconfined phase).

In the present case, the effect of the one-loop thermal mass (21) on the Gribov restriction will be considered by setting $m=m(T)$, and it will be shown that there exist critical temperatures corresponding to the above three different regimes. It is worth noting that the inclusion of such a one-loop mass is fundamental in order to obtain these different phases.

\subsection{Thermal gap equation}

As it has been already discussed in the introduction of this manuscript, two important requirements for the consistency of the analysis are the following. Firstly, the finitetemperature gap equation should have, when the temperature is low enough, solutions close to the zero-temperature one, describing confined gluons. Secondly, when the temperature is high enough, the gap equation should have no solution, which describes propagating gluons.

As is well known, these conditions are not easy to satisfy $[35,36]$. In the present paper, we will include the one-loop perturbative corrections both in the running coupling (see Sect. 4) and in the field propagators (since the crucial role of the one-loop mass is well known: see [37] and references therein). In order to write down the gap equation for the finite-temperature case, we apply the prescription (16)-(23) and take the infinite spatial volume limit

$\frac{1}{V} \sum_{q} \rightarrow T \sum_{n} \int \frac{\mathrm{d}^{3} q}{(2 \pi)^{3}}$.

Finally, replacing the thermal gluon mass (21), we obtain the following thermal gap equation:

$$
\frac{3 N g^{2} T}{8 \pi^{2}} \sum_{n} \int_{0}^{\Lambda} \frac{r^{2} \mathrm{~d} r}{\left(r^{2}+\omega_{n}^{2}\right)^{2}+\frac{N g^{2} T^{2}}{9}\left(r^{2}+\omega_{n}^{2}\right)+\gamma^{4}}=1,
$$

where we have adopted polar coordinates, integrated over angular variables, and we defined a radial integration limit $\Lambda$, which corresponds to an ultraviolet cutoff. Let us note that we have neglected the second term of (23), as it goes to zero for an infinite spatial volume.

Defining the dimensionless variables

$$
\begin{aligned}
& R=\frac{r}{\Lambda}, \quad \lambda=\frac{2 \pi T}{\Lambda} \\
& \theta_{n}=\frac{\omega_{n}}{\Lambda}=n \lambda, \quad \Gamma=\frac{\gamma}{\Lambda},
\end{aligned}
$$

the thermal gap equation can be rewritten as

$$
\frac{3 N g^{2} \lambda}{16 \pi^{3}} \sum_{n} \int_{0}^{1} \frac{R^{2} \mathrm{~d} R}{\left(R^{2}+\theta_{n}^{2}\right)^{2}+\frac{N g^{2} \lambda^{2}}{36 \pi^{2}}\left(R^{2}+\theta_{n}^{2}\right)+\Gamma^{4}}=1 .
$$

The sum over all dimensionless Matsubara frequencies $\theta_{n}$ can be carried out analytically (see Appendix), leading to

$$
\begin{aligned}
S(R, \lambda, \Gamma)= & \sum_{n} \frac{1}{\left(R^{2}+\theta_{n}^{2}\right)^{2}+\frac{N g^{2} \lambda^{2}}{36 \pi^{2}}\left(R^{2}+\theta_{n}^{2}\right)+\Gamma^{4}} \\
= & \frac{\pi}{2 \lambda \sqrt{\frac{N^{2} g^{4} \lambda^{4}}{72^{2} \pi^{4}}-\Gamma^{4}}} \\
& \times\left(\frac{\cot h\left(\frac{\pi}{\lambda} \sqrt{R^{2}+\frac{N g^{2} \lambda^{2}}{72 \pi^{2}}-\sqrt{\frac{N^{2} g^{4} \lambda^{4}}{72^{2} \pi^{4}}-\Gamma^{4}}}\right)}{\sqrt{R^{2}+\frac{N g^{2} \lambda^{2}}{72 \pi^{2}}-\sqrt{\frac{N^{2} g^{4} \lambda^{4}}{72^{2} \pi^{4}}-\Gamma^{4}}}}\right. \\
& \left.-\frac{\cot h\left(\frac{\pi}{\lambda} \sqrt{R^{2}+\frac{N g^{2} \lambda^{2}}{72 \pi^{2}}+\sqrt{\frac{N^{2} g^{4} \lambda^{4}}{72^{2} \pi^{4}}-\Gamma^{4}}}\right)}{\sqrt{R^{2}+\frac{N g^{2} \lambda^{2}}{72 \pi^{2}}+\sqrt{\frac{N^{2} g^{4} \lambda^{4}}{72^{2} \pi^{4}}-\Gamma^{4}}}}\right)
\end{aligned}
$$

and the gap equation takes the form

$\frac{3 N g^{2} \lambda}{16 \pi^{3}} \int_{0}^{1} \mathrm{~d} R R^{2} S(R, \lambda, \Gamma)=1$,

which defines $\gamma$ as a function of $\lambda$,

$\gamma=\Lambda \Gamma(\lambda)$

\section{The three regimes}

As we have shown in Sect. 3, the effective gluon propagator (24) can lead to three different regimes for gluons depending on the value of the thermal mass $m_{p l}(T)$, which in turn depends on the temperature $T$. These three phases can be associated to two transition temperatures. In this section we present the numerical analysis of the gap equation (32) for 
Fig. 1 Plot of the surface $F$ for different values of $\lambda$ and $\Gamma$. The intersection with the plane $Y=1$ occurs for $\lambda$ below the critical value $\lambda_{c}^{(1)}=1.4$

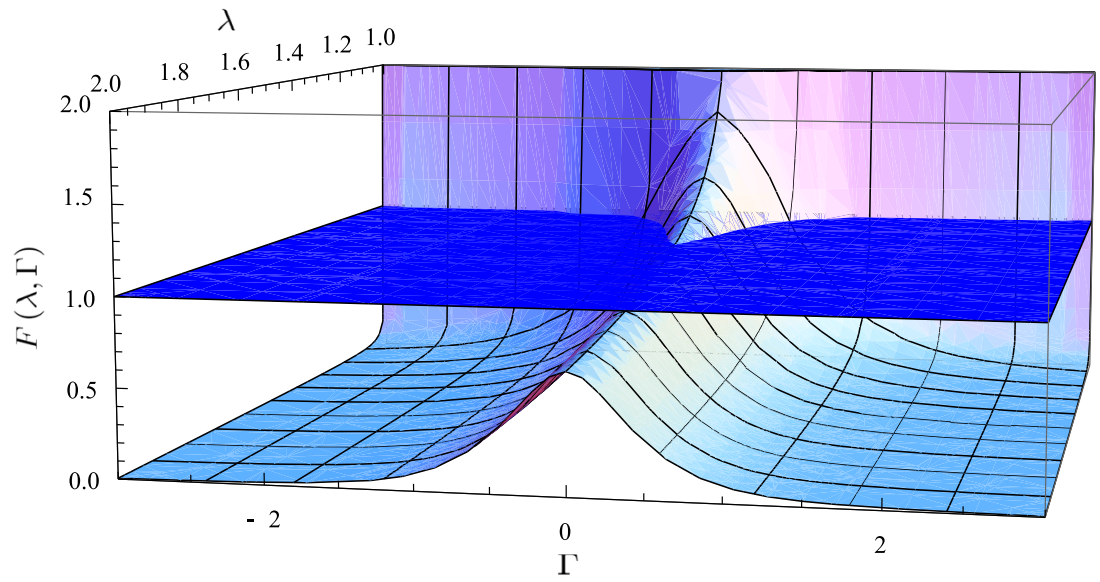

Fig. 2 Plot of $F$ as a function of $\Gamma$, for $\lambda=1.2,1.4$ and 2.0

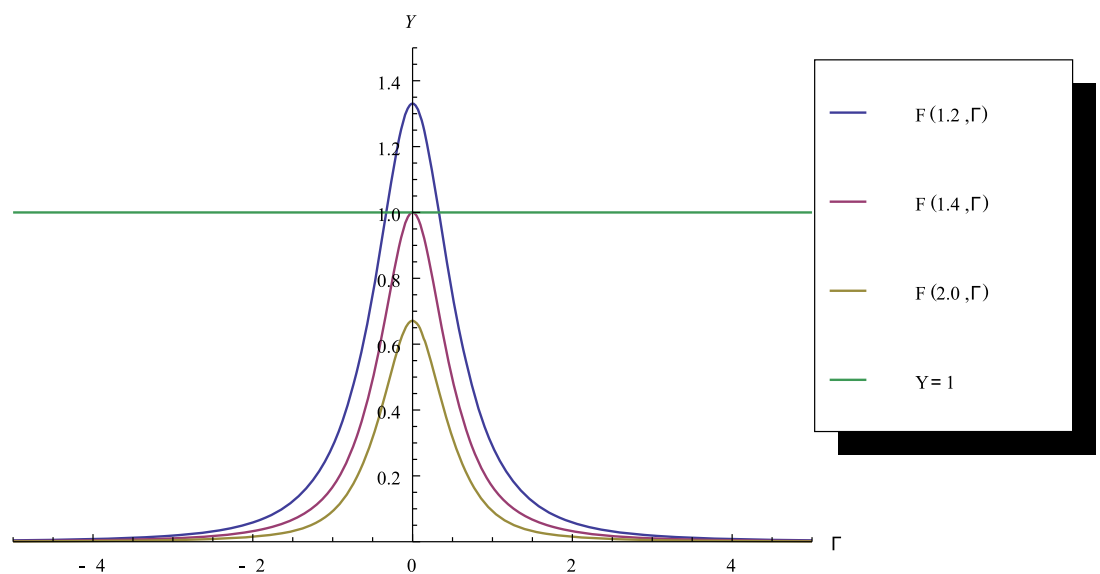

QCD $(N=3)$ in the high-temperature regime and subsequently we study a possible infrared continuation.

\subsection{High-temperature running coupling}

Let us consider the thermal gap equation in the limit of high temperatures $T>>1$. In finite-temperature QCD, the oneloop running coupling depends on the temperature $T$ (or, in our case, on $\lambda$ ) as $[35,43]$

$g^{2}(\lambda)=\frac{8 \pi^{2}}{11 \ln \left(\frac{2 \pi T}{\Lambda_{\mathrm{QCD}}}\right)}=\frac{8 \pi^{2}}{11 \ln (\alpha \lambda)}$,

where we have defined the ratio between the cutoff $\Lambda$ and the energy scale $\Lambda_{\mathrm{QCD}}$ as

$\alpha \equiv \frac{\Lambda}{\Lambda_{\mathrm{QCD}}}$

For the left hand side of (32), we define the function

$F(\lambda, \Gamma)=\frac{9 g^{2} \lambda}{16 \pi^{3}} \int_{0}^{1} \mathrm{~d} R R^{2} S(R, \lambda, \Gamma)$.
Then the solution for the gap equation corresponds to the intersection of the curves $Y=F(\lambda, \Gamma)$ with $Y=1$.

In order to obtain the qualitative behavior for the solutions, we will consider $\alpha=1$ in the analysis below (as will be explained later on, the qualitative behavior of the gluon propagator does not depend on the value of $\alpha$ ). From Fig. 1, we see that the existence of solution depends on the temperature. In fact, the intersection occurs for $\lambda$ 's below a critical value $\lambda_{c}^{(1)}=1.4$; see Fig. 2 . This corresponds to a phase transition at temperature

$\frac{T_{c}^{(1)}}{\Lambda_{\mathrm{QCD}}}=0.22$.

For $T>T_{c}^{(1)}$ there is no solution for the gap equation (32). In this case the only consistent choice for the Gribov parameter is $\gamma=0$, indicating that this regime represents the free phase. On the other hand, for $T<T_{c}^{(1)}$, there is a solution for the gap equation, which define the Gribov parameter $\gamma$. Therefore, as is shown in Fig. $3, \Gamma$ decreases as $\lambda$ increases and vanishes for $\lambda^{(1)}=1.4$.

Even though for $\lambda<1.4$ there is a solution for the gap equation, the propagator is still not completely confining. As we saw in Sect. 3, depending on the sign of the discriminant 


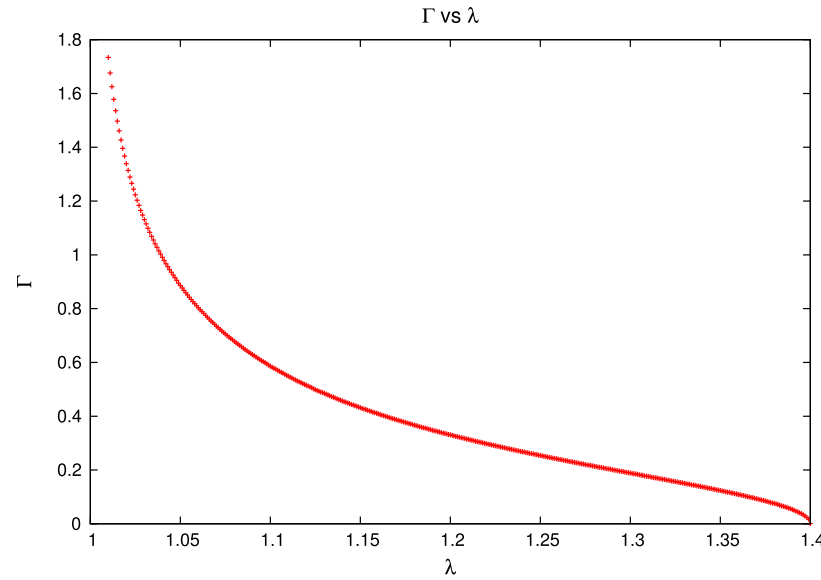

Fig. 3 Plot $\Gamma$ vs. $\lambda$. At $\lambda^{(1)} \sim 1.4$ there exists a phase transition from a deconfined phase to a semi-confined one, which corresponds $\frac{T_{c}^{1}}{\Lambda}=0.22$

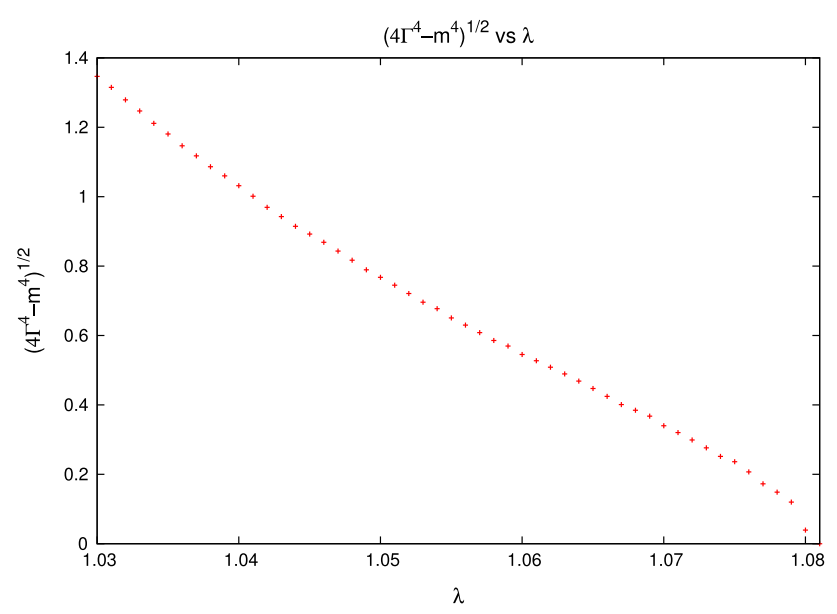

Fig. 4 Plot $\sqrt{4 \Gamma^{4}-m^{4}}$ vs. $\lambda$. At $\lambda^{(2)} \sim 1.08$ there exists a phase transition from a semi-confined phase to a confined one, which corresponds $\frac{T_{c}^{2}}{\Lambda}=0.17$

in (25), a partial or total confinement can take place. In this case, the change of sign in (25) occurs for $\lambda_{c}^{(2)}=1.08$ (see Fig. 4), which corresponds to

$\frac{T_{c}^{(2)}}{\Lambda_{\mathrm{QCD}}}=0.17$.

Hence, two phase transitions are found as the temperature decreases: a deconfined/partially deconfined/confined phase transition at $T_{c}^{(1)}$ and a partially deconfined/confined phase transition at $T_{c}^{(2)}$. In the intermediate phase, only one degree of freedom of the gluon is physical, as discussed in Sect. 3.2.

\subsection{Infrared continuation}

In order to extend the analysis of the previous subsection to the low-temperature regime, we need a prescription to extend the definition (34) for $\lambda<1$.
A way to extend the running coupling to the infrared regime in zero-temperature QCD has been developed in [44] in the framework of quark-antiquark potentials by adding a non-perturbative contribution to the Wilson loop. In the finite-temperature case, the analog extension reads

$g^{2}\left(g_{0}, \lambda\right)=\frac{g_{0}^{2}}{1+\frac{11}{16 \pi^{2}} g_{0}^{2} \ln \left(1+\alpha^{2} \lambda^{2}\right)}$.

This expression reduces to (34) for large $\lambda$ but, in the limit $\lambda \rightarrow 0$ the running coupling reduces to the bare coupling constant $g_{0}$

$g^{2} \underset{\lambda \rightarrow 0}{\longrightarrow} g_{0}^{2}$

This choice is also consistent with the fact that the thermal gluon mass (21) must vanish as $T$ goes to zero

$m_{p l}^{2} \underset{T \rightarrow 0}{\longrightarrow} 0$

which is a necessary requirement to reduce (23)-(12) in this limit and to connect consistently with the standard $T=0$ results [10]. Let us note that for large $g_{0}$ the behavior of $g\left(g_{0}, \lambda\right)$ becomes insensible to small variations of $g_{0}$ itself; see Fig. 5. This is consistent with the fact that in quantum field theory bare quantities are infinite but unobservable and they need to be renormalized.

Replacing the expression (39) (with $\alpha=1$ ) in the gap equation (32), the left hand side takes the form

$G\left(g_{0}, \lambda, \Gamma\right)=\frac{9 g^{2} \lambda}{16 \pi^{3}} \int_{0}^{1} \mathrm{~d} R R^{2} S\left(R, g_{0}, \lambda, \Gamma\right)$,

where $S\left(R, g_{0}, \lambda, \Gamma\right)$ is obtained replacing (39) in (31). Then, the solution for the gap equation again corresponds to the intersection of the curves $Y=G\left(g_{0}, \lambda, \Gamma\right)$ and $Y=1$, whose existence depends on $\lambda$ (see Fig. 6).

Similarly to the previous subsection, we find two phase transitions. Choosing $g_{0}=1,000$, the deconfined/partially deconfined phase transition occurs for the critical value $\lambda_{c}^{(1)}=1.17$ (see Figs. 7, 8), which corresponds to

$\frac{T_{c}^{(1)}}{\Lambda_{\mathrm{QCD}}}=0.19$,

while the semi-confinement/confinement phase transition now occurs for $\lambda_{c}^{(2)}=0.81$ (see Fig. 9), i.e.,

$\frac{T_{c}^{(2)}}{\Lambda_{\mathrm{QCD}}}=0.13$

The results obtained with the prescription (39) are very similar to the ones obtained in the previous subsection. It is important to note that the qualitative behavior of the solution of the gap equation and the gluon propagator does not dependon the value of $\alpha$ in the definition 
Fig. 5 Plot of the running coupling $g$ as a function of $g_{0}$ and $\lambda$. For $g_{0}$ large, $g$ becomes almost insensible to small variations of $g_{0}$
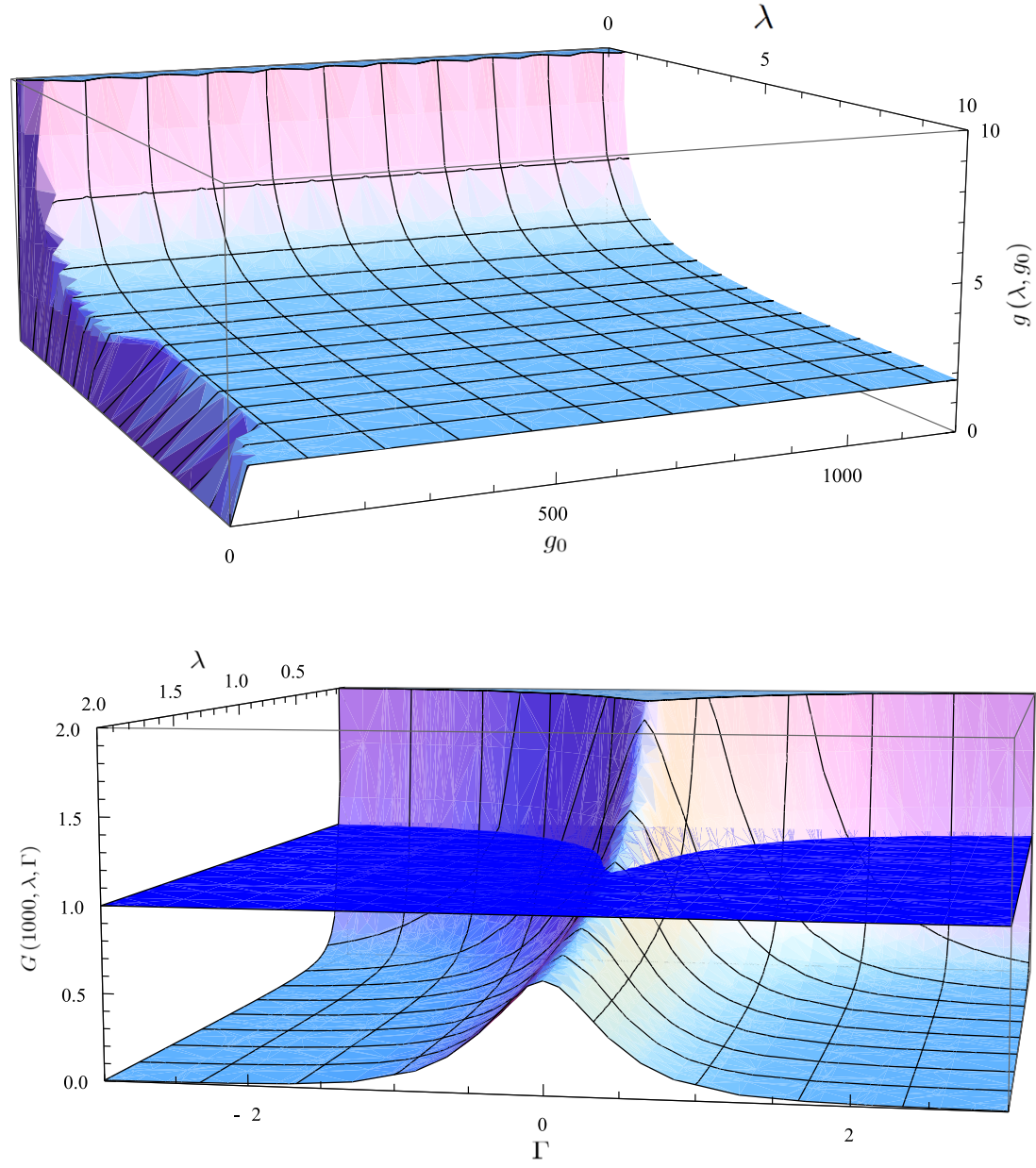

Fig. 6 Plot of the surface $F$ for different values of $\lambda$ and $\Gamma$. The intersection with the plane $Y=1$ occurs for $\lambda$ below a critical value $\lambda_{c}^{(1)}=1.17$

Fig. 7 Plot of $F$ as a function of $\Gamma$, for $\lambda=0.8,1.17$ and 2.5

(35). As we can see in Table 1, the greater the value of $\alpha$ that we consider in the analysis (i.e. the greater the cutoff $\Lambda$ compared with QCD scale $\Lambda_{\mathrm{QCD}}$ ), the greater will be the numerical values for the critical temperatures for the phase transitions. Hence, the fact that the integration cutoff $\Lambda$ is much higher than the QCD scale $\Lambda_{\mathrm{QCD}}$ implies that the critical temperatures obtained with this method, when considering a more realistic ratio between this quantities, will be greater than the values obtained in this section.

On the other hand, in our analysis we have considered only gluon dynamics (without quarks). In [29,30] it has been found that the value for the energy scale $\Lambda_{\mathrm{QCD}}$ that must be considered depends on the numbers of flavors that are included in the analysis and there have been found different values for $\frac{T_{c}}{\Lambda_{\mathrm{QCD}}}$ depending on these considerations. 


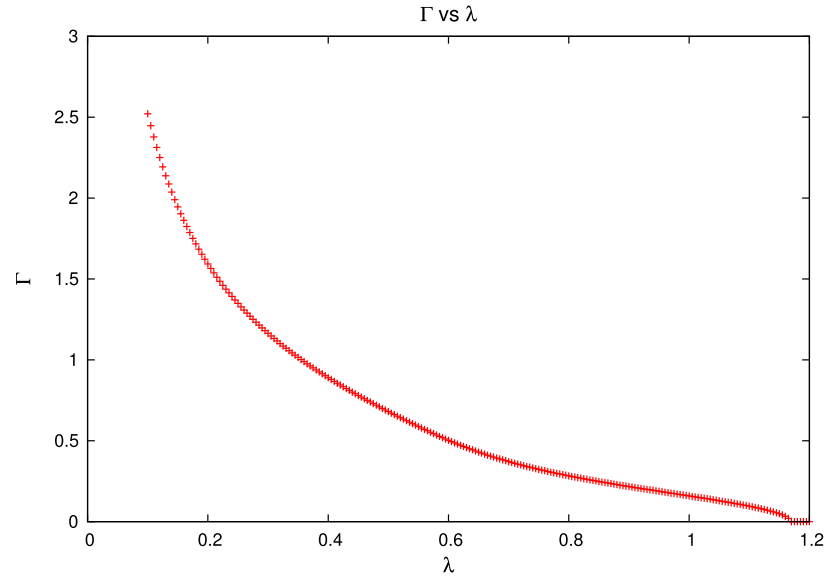

Fig. 8 Plot $\Gamma$ vs. $\lambda$. At $\lambda^{(1)} \sim 1.17$ there exists a phase transition from a deconfined phase to a semi-confined one, which corresponds to $\frac{T_{c}^{(1)}}{\Lambda}=0.19$

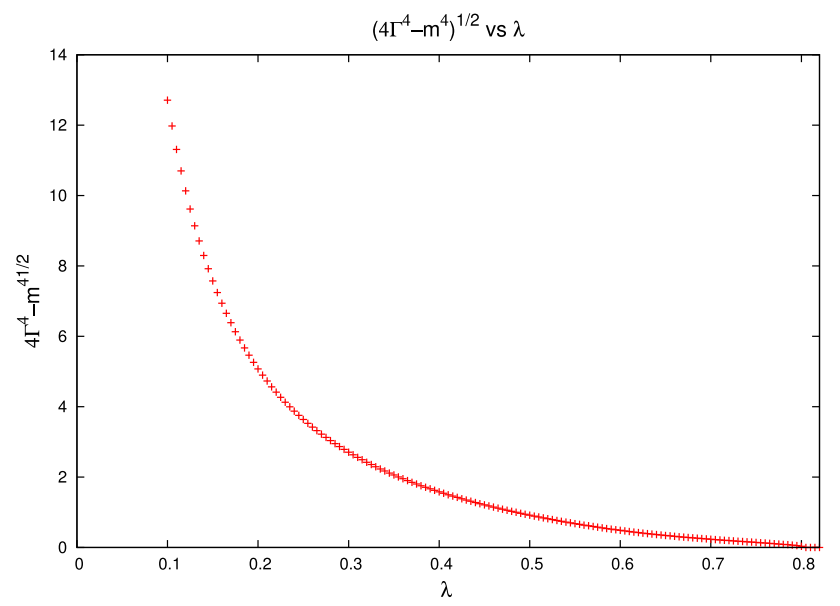

Fig. 9 Plot $\sqrt{4 \Gamma^{4}-m^{4}}$ vs. $\lambda$. At $\lambda^{(2)} \sim 0.81$ there exists a phase transition from a semi-confined phase to a confined one, which corresponds to $\frac{T_{c}^{(2)}}{\Lambda}=0.13$

Table 1 Critical temperatures $\frac{T_{c}^{(1)}}{\Lambda_{\mathrm{QCD}}}$ and $\frac{T_{c}^{(2)}}{\Lambda_{\mathrm{QCD}}}$ for different values of $\alpha$. Here, $g_{\mathrm{HT}}$ and $g_{\mathrm{IC}}$ correspond to the running coupling at high temperature (34) and its infrared continuation (39), respectively

\begin{tabular}{llllll}
\hline$\alpha$ & $g_{\mathrm{HT}}$ & & & \multicolumn{2}{l}{$g_{\mathrm{IC}}$} \\
\cline { 2 - 5 } & $\frac{T_{c}^{(1)}}{\Lambda_{\mathrm{QCD}}}$ & $\frac{T_{c}^{(2)}}{\Lambda_{\mathrm{QCD}}}$ & & $\frac{T_{c}^{(1)}}{\Lambda_{\mathrm{QCD}}}$ & $\frac{T_{c}^{(2)}}{\Lambda_{\mathrm{QCD}}}$ \\
\hline 1 & 0.223 & 0.172 & 0.186 & 0.128 \\
10 & 0.437 & 0.331 & 0.414 & 0.301 \\
100 & 0.758 & 0.558 & 0.752 & 0.540 \\
\hline
\end{tabular}

\section{Discussion and future developments}

In this paper it has been shown that the semi-classical Gribov approach applied to finite-temperature YM-theory is consistent with the presence of a confined/deconfined phase transi- tion. This is reflected in the fact that the existence of solutions of the Gribov gap equation depends on the temperature.

A key ingredient for the consistent description of these different regimes is the inclusion of a mass term in the gluon propagator, which comes from the one-loop corrections to the theory. Indeed, if the mass term is not taken into account, there are no critical temperatures at all and one would be left with confined gluons at all the temperatures. Furthermore, to include one-loop corrections is consistent with the fact that the thermal mass (21) causes gluon deconfinement [37].

In order to be able to study the low-temperature limit, we have introduced a modified running coupling $g$, which interpolates between the standard perturbative result in the ultraviolet regime and a constant (in principle infinite but unobservable) for the infrared regime. It is worth to note that this modification has been considered only for consistency, as it allows the gluon thermal mass to go to zero for low temperature, but the presence of these phase transitions does not depend on this fact. Indeed, the same qualitative behavior for the gluon propagator was obtained when considering the standard one-loop running coupling (34), and, furthermore, it can be shown that phase transitions are also present if only a constant coupling is considered in the whole analysis. We stress that when the Gribov semi-classical method is implemented at zero temperature but with a non-trivial Higgs field (see $[45,46])$, the phase diagram turns out to be very close to the one obtained in the present paper in agreement with the Fradkin-Shenker theorem [47].

In this paper we have considered the scaling solution, in which the gluon propagator (13) vanishes and the ghost propagator (14) blows up as $\frac{1}{q^{4}}$ in the infrared limit $q \rightarrow 0$. On the other hand, it is clear by now that the decoupling solution (where the gluon propagator goes to a constant in the infrared limit while the ghost propagator has a free-like behavior) is the relevant one ${ }^{3}[49,50]$. The decoupling solution has a strong lattice support [51-54] and can be obtained analytically within the refined Gribov-Zwanziger theory by including some condensates [21-23]. It would certainly be of interest to study the refined Gribov-Zwanziger approach at finite temperature. However, as this theory includes extra ghost fields necessary to express the action in a local form, the main technical problem when passing to the finitetemperature formalism is to determine the boundary conditions that these extra fields must satisfy. This issue is under investigation and we hope to come back to this point in the future.

An interesting result of this paper is the appearance of an intermediate regime in between confined and free regimes, in which only one of the two gluonic degrees of freedom is

\footnotetext{
${ }^{3}$ In Ref. [48] the effect of Gribov horizon in the Schwinger-Dyson equations has been studied obtaining both the scaling and the decoupled solution.
} 
physical, while the other one does not belong to the physical spectrum. In this sense, this new regime captures traces of both confined and deconfined regimes. Hence, this scenario could be interpreted as a partial deconfinement or a semiQGP phase, which has been studied in [32-34]. Regimes of this kind can appear when studying QGP by different methods. In fact, in a very interesting paper [31] the phase transition in hot QCD is analyzed in the context of electrically and magnetically charged quasi-particles, where the confined regime corresponds to a magnetically dominated and electrically confined region, while the free regime is described by a magnetically strongly correlated and electrically dominated region. In between these regimes, a "post-confined" region is found, where electrically charged excitations are strongly correlated, which can also be interpreted as a partial deconfinement. It is reassuring that, even though this method is quite different from our approach, the qualitative results are in agreement with ours, as far as the presence of an intermediate regime is concerned.

Despite the fact that pure Yang-Mills theory is interesting in itself, the inclusion of quarks is important in order to obtain a more realistic model. This point requires a careful analysis since, as has been shown in [55-58] (see also [59]), the quark propagator develops complex poles at the non-perturbative level in the same way as the gluon propagator does after implementing the Gribov restriction. According to the analysis for a propagator with complex poles given in Sect. 3.2, the fact that the quark and gluon propagators share this feature in the infrared strongly suggests that there could exist an intermediate quark regime as well. On the other hand, the thermodynamics of quark models with complex mass poles have been studied in [60], and it would be interesting to follow its lines when adding quarks to the Gribov-Zwanziger theory. The study of the equation of state for gluons, both in the semi-classical Gribov approach and the Gribov-Zwanziger theory, as well as the inclusion of quarks, presents several technical difficulties at the analytical and numerical level, and they are currently under investigation.

Another important subject in the understanding of deconfinement, which is also the aim of a future work, is the order of the phase transition. In order to formally associate each regime of Sect. 4 with a phase of a gluon plasma and prove rigorously that the critical temperatures that we have found determine a phase transition, an order parameter must be introduced. The natural choice for the order parameter in the finite-temperature formalism is the Polyakov loop, and its computation for the intermediate phase found in Sect. 3.2 would shed light on its relation with partial deconfinement and semi-QGP. On the other hand, as has been explained in [60], the Polyakov loop is very useful when dealing with nontrivial thermodynamics arising in the presence of complex.
Acknowledgments We wish to thank David Dudal and Silvio Sorella for very useful comments. This work has been funded by the Fondecyt Grants No. 1120352. The Centro de Estudios Científicos (CECs) is funded by the Chilean Government through the Centers of Excellence Base Financing Program of CONICYT. P.P. thanks the Physique Théorique et Mathématique group of the Université Libre de Bruxelles, the International Solvay Institutes and the Faculty of Mathematics and Physics of Charles University in Prague for the kind hospitality during the development of this work. P.P. is supported by grants from Universidad Andrés Bello. P.S.-R. is supported by grants from BECAS CHILE, Comisión Nacional de Investigación Científica y Tecnológica CONICYT and from Universidad de Concepción, Chile.

Open Access This article is distributed under the terms of the Creative Commons Attribution License which permits any use, distribution, and reproduction in any medium, provided the original author(s) and the source are credited.

Funded by $\mathrm{SCOAP}^{3}$ / License Version CC BY 4.0.

\section{Appendix: Sum over Matsubara frequencies}

Let us consider the gap equation (30)

$$
\frac{3 N g^{2} \lambda}{16 \pi^{3}} \sum_{n} \int_{0}^{1} \frac{R^{2} \mathrm{~d} R}{\left(R^{2}+\theta_{n}^{2}\right)^{2}+\frac{N g^{2} \lambda^{2}}{36 \pi^{2}}\left(R^{2}+\theta_{n}^{2}\right)+\Gamma^{4}}=1,
$$

and let us compute the following sum over the dimensionless Matsubara frequencies $\theta_{n}$ :

$$
\sum_{n} \frac{1}{\left(R^{2}+\theta_{n}^{2}\right)^{2}+\frac{N g^{2} \lambda^{2}}{36 \pi^{2}}\left(R^{2}+\theta_{n}^{2}\right)+\Gamma^{4}}=\sum_{n} \frac{1}{P\left(n^{2}\right)},
$$

where

$$
\begin{aligned}
& P(x)=\lambda^{4}\left(x+a_{-}\right)\left(x+a_{+}\right), \\
& a_{ \pm}=\frac{R^{2}}{\lambda^{2}}+\frac{N g^{2}}{72 \pi^{2}} \pm \sqrt{\frac{N^{2} g^{4}}{72^{2} \pi^{4}}-\frac{\Gamma^{4}}{\lambda^{4}} .}
\end{aligned}
$$

Using algebraic manipulations, we can write (43) as

$$
\sum_{n} \frac{1}{P\left(n^{2}\right)}=\frac{1}{\lambda^{4}} \frac{1}{a_{+}-a_{-}} \sum_{n}\left(\frac{1}{n^{2}+a_{-}}-\frac{1}{n^{2}+a_{+}}\right) .
$$

Then, using the residue theorem applied to the sum series,

$$
\sum_{n=-\infty}^{\infty} f(z)=-\sum \operatorname{res}[\pi \cot (\pi z) f(z)]
$$

we obtain for (46)

$$
\begin{aligned}
\sum_{n} \frac{1}{P\left(n^{2}\right)}= & \frac{1}{\lambda^{4}} \frac{1}{a_{+}-a_{-}}\left(\frac{\pi \cot h\left(\pi \sqrt{a_{-}}\right)}{\sqrt{a_{-}}}\right. \\
& \left.-\frac{\pi \cot h\left(\pi \sqrt{a_{+}}\right)}{\sqrt{a_{+}}}\right) .
\end{aligned}
$$


Defining $S(R, \lambda, \Gamma)=\sum_{n} \frac{1}{P\left(n^{2}\right)}$ and using (45) we obtain (31):

$$
\begin{aligned}
S(R, \lambda, \Gamma)= & \frac{\pi}{2 \lambda \sqrt{\frac{N^{2} g^{4} \lambda^{4}}{72^{2} \pi^{4}}-\Gamma^{4}}} \\
& \times\left(\frac{\operatorname{coth}\left(\frac{\pi}{\lambda} \sqrt{R^{2}+\frac{N g^{2} \lambda^{2}}{72 \pi^{2}}-\sqrt{\frac{N^{2} g^{4} \lambda^{4}}{72^{2} \pi^{4}}-\Gamma^{4}}}\right)}{\sqrt{R^{2}+\frac{N g^{2} \lambda^{2}}{72 \pi^{2}}-\sqrt{\frac{N^{2} g^{4} \lambda^{4}}{72^{2} \pi^{4}}-\Gamma^{4}}}}\right. \\
& \left.-\frac{\operatorname{coth}\left(\frac{\pi}{\lambda} \sqrt{\left.R^{2}+\frac{N g^{2} \lambda^{2}}{72 \pi^{2}}+\sqrt{\frac{N^{2} g^{4} \lambda^{4}}{72^{2} \pi^{4}}-\Gamma^{4}}\right)}\right.}{\sqrt{R^{2}+\frac{N g^{2} \lambda^{2}}{72 \pi^{2}}+\sqrt{\frac{N^{2} g^{4} \lambda^{4}}{72^{2} \pi^{4}}-\Gamma^{4}}}}\right)
\end{aligned}
$$

and the thermal gap equation (30) takes the form

$\frac{3 N g^{2} \lambda}{16 \pi^{3}} \int_{0}^{1} \mathrm{~d} R R^{2} S(R, \lambda, \Gamma)=1$.

\section{References}

1. D.J. Gross, F. Wilczek, Phys. Rev. Lett. 30, 1343 (1973)

2. H.D. Politzer, Phys. Rev. Lett. 30, 1346 (1973)

3. V.N. Gribov, Nucl. Phys. B 139, 1 (1978)

4. I.M. Singer, Commun. Math. Phys. 60, 7 (1978)

5. R. Jackiw, I. Muzinich, C. Rebbi, Phys. Rev. D 17, 1576 (1978)

6. F. Canfora, A. Giacomini, J. Oliva, Phys. Rev. D 82, 045014 (2010)

7. A. Anabalón, F. Canfora, A. Giacomini, J. Oliva, Phys. Rev. D 83, $064023(2011)$

8. F. Canfora, A. Giacomini, J. Oliva, Phys. Rev. D 84, 105019 (2011)

9. M. de Cesare, G. Esposito, H. Ghorbani, Phys. Rev. D 88, 087701 (2013)

10. R.F. Sobreiro, S.P. Sorella, Introduction to the Gribov ambiguities in Euclidean Yang-Mills theories. arXiv:hep-th/0504095

11. F. Canfora, P. Salgado-Rebolledo, Phys. Rev. D 87, 045023 (2013)

12. G.F. Dell'Antonio, D. Zwanziger, Nucl. Phys. B 326, 333 (1989)

13. D. Zwanziger, Nucl. Phys. B323, 513 (1989)

14. D. Zwanziger, Nucl. Phys. B399, 477 (1993)

15. P. van Baal, Nucl. Phys. B369, 25 (1992)

16. N. Vandersickel, D. Zwanziger, The Gribov problem and QCD dynamics. Phys. Rept. 520, 175 (2012). 1202.1491 [hep-th]

17. M. Maggiore, M. Schaden, Phys. Rev. D 50, 6616 (1994)

18. J.A. Gracey, JHEP 0605, 052 (2006)

19. D. Dudal, R.F. Sobreiro, S.P. Sorella, H. Verschelde, Phys. Rev. D 72, 014016 (2005)

20. D. Dudal, S.P. Sorella, N. Vandersickel, H. Verschelde, Phys. Rev. D 77, 071501 (2008)

21. S.P. Sorella, D. Dudal, M.S. Guimaraes, N. Vandersickel, PoS FACESQCD, 022 (2010). arXiv:1102.0574 [hep-th]

22. D. Dudal, J.A. Gracey, S.P. Sorella, N. Vandersickel, H. Verschelde, Phys. Rev. D 78, 065047 (2008). arXiv:0806.4348 [hep-th]

23. D. Dudal, M.S. Guimaraes, S.P. Sorella, Phys. Rev. Lett. 106, 062003 (2011)

24. F. Canfora, L. Rosa, Phys. Rev. D 88, 045025 (2013)

25. M. Le Bellac, Thermal Field Theory (Cambridge University Press, Cambridge, 2000)

26. K. Yagi, T. Hatsuda, Y. Miake, Quark-Gluon Plasma: From Big Bang to Little Bang (Cambridge University Press, Cambridge, 2005)
27. N.K. Glendenning, Compact Stars: Nuclear Physics, Particle Physics, and General Relativity (Springer, New York, 1997)

28. J. Kapusta, B. Muller, J. Rafeslki, Quark-Gluon Plasma: Theoretical Foundations (Elseiver, Amsterdam, 2003)

29. C.P. Korthals Altes, in Symmetries and Quasi-Particles in Hot $Q C D$. ed. by A. Zichichi. Proceedings of the International School of Subnuclear Physics (Singapore: World Scientific Publishing, 2004)

30. S. Gupta, Phys. Rev. D 64, 034507 (2001)

31. J. Liao, E. Shuryak, Phys. Rev. C 75, 054907 (2007). arXiv:0611131 [hep-ph]

32. R.D. Pisarski, Nucl. Phys. B (Proc. Suppl.) 195, 157198 (2009)

33. Y. Hidaka, R.D. Pisarski, Phys. Rev. D 78, 071501 (2008). arXiv:0803.0453 [hep-ph]

34. R.D. Pisarski, K. Kashiwa, V. Skokov, Nucl. Phys. A 904-905, 973c (2013). arXiv:1305.0767 [hep-ph]

35. D. Zwanziger, Phys. Rev. D 76, 12504 (2007)

36. K. Fukushima, N. Su, Phys. Rev. D 88, 076008 (2013)

37. P.B. Arnold, Int. J. Mod. Phys. E16, 255 (2007)

38. U. Reinosa, J. Serreau, M. Tissier, N. Wschebor, arXiv:1311.6116 [hep-th]

39. R. Alkofer, L. von Smekal, Phys. Rept. 353, 281 (2001). [hep$\mathrm{ph} / 0007355]$

40. M.E. Peskin, D.V. Schroeder, An Introduction to Quantum Field Theory. (Perseus Books Publishing L.L.C., New York, 1995)

41. J.I. Kapusta, C. Gale, Finite-Temperature Field Theory: Principles and Applications (Cambridge University Press, Cambridge, 2006)

42. R.F. Sobreiro, S.P. Sorella, D. Dudal, H. Verschelde, Phys. Lett. B 590, 265-272 (2004)

43. S. Huang, Nucl. Phys. B 438, 54 (1995)

44. G.M. Prosperi, M. Raciti, C. Simolo, Prog. Part. Nucl. Phys. 58, 387 (2007)

45. M.A.L. Capri, D. Dudal, A.J. Gomez, M.S. Guimaraes, I.F. Justo, S.P. Sorella, D. Vercauteren, Phys. Rev. D 88, 085022 (2013)

46. M.A.L. Capri, D. Dudal, A.J. Gomez, M.S. Guimaraes, I.F. Justo, S.P. Sorella, Eur. Phys. J. C 73, 2346 (2013)

47. E. Fradkin, S. Shenker, Phys. Rev. D 19, 3682 (1979)

48. K.-I. Kondo, Decoupling and scaling solutions in Yang-Mills theory with the Gribov horizon. arXiv:0909.4866 [hep-th]

49. Ph. Boucaud, J.P. Leroy, A. Le Yaouanc, J. Micheli, O. Pen e, J. Rodriguez-Quintero, JHEP 06, 099 (2008). arXiv:0803.2161[hepph]

50. Ph. Boucaud, J.P. Leroy, A. Le Yaouanc, J. Micheli, O. Pen e, J. Rodriguez-Quintero, JHEP 06, 012 (2008). arXiv:0801.2721[hep$\mathrm{ph}]$

51. A. Cucchieri, T. Mendes, PoS LAT2007, 297 (2007)

52. I.L. Bogolubsky, E.M. Ilgenfritz, M. Muller-Preussker, A. Sternbeck, PoS LAT2007, 290 (2007)

53. A. Cucchieri, T. Mendes, Phys. Rev. Lett. 100, 241601 (2008). arXiv:0712.3517 [hep-lat]

54. A. Cucchieri, T. Mendes, Phys. Rev. D 78, 094503 (2008). arXiv:0804.2371 [hep-lat]

55. S. Furui, H. Nakajima, Phys. Rev. D 73, 074503 (2006)

56. M.B. Parappilly, P.O. Bowman, U.M. Heller, D.B. Leinweber, A.G. Williams, J.B. Zhang, Phys. Rev. D 73, 054504 (2006)

57. G. Burgio, M. Schrock, H. Reinhardt, M. Quandt, Phys. Rev. D 86, 014506 (2012)

58. E. Rojas, J.P.B.C. de Melo, B. El-Bennich, O. Oliveira, T. Frederico. arXiv:1306.3022 [hep-ph]

59. D. Dudal, M.S. Guimaraes, L.F. Palhares, S.P. Sorella, From QCD to a dynamical quark model: construction and some meson spectroscopy. arXiv:1303.7134 [hep-ph]

60. S. Benić, D. Blaschke, M. Buballa, Phys. Rev. D 86, 074002 (2012). arXiv:1206.6582 [hep-ph] 